\title{
Some observations on the activity of terminal constraints in linear MPC
}

\author{
Moritz Schulze Darup and Mark Cannon
}

\begin{abstract}
We analyze the activity of terminal constraints in stabilizing MPC for linear systems with state and input constraints. In particular, we show that the set of initial states for which the terminal constraints are inactive (or weakly active), is always compact but may or may not be convex. In addition, we illustrate that the set where terminal constraints are (strongly) active may be nonempty, even for arbitrarily long prediction horizons. This observation is important for the design of stabilizing MPC schemes without terminal constraints.
\end{abstract}

\section{INTRODUCTION}

It is well-known that, for linear constrained systems, there exists a close relation between constrained linear-quadratic regulation (CLQR, see, e.g., [1]-[3]) and model predictive control (MPC) with guaranteed stability. In fact, stabilizing MPC schemes usually build on terminal sets where the solution to the CLQR problem is explicitly known (see, e.g., [4] or [5]). Nevertheless, there also exist stabilizing MPC approaches that do not take terminal sets into account (see, e.g., [6]). These approaches require, however, a suitable choice of the prediction horizon such that optimal trajectories are implicitly guaranteed to terminate in a "safe" region around the origin. In other words, the prediction horizon has to be chosen such that the inclusion of a terminal set in the optimal control problem (OCP) would never result in strongly active terminal contraints (for a certain set of initial states, see [6, Thm. 13] for details).

In this paper, we study the activity of terminal constraints in linear MPC. In particular, we analyze the relation between the feasible set of MPC schemes with terminal constraints and the set of states where these constraints are (strongly) active (referred to as the set of (strong) activity (cf. [8])). The paper is organized as follows. We specify the problem of interest and state some preliminaries in Section II. The main result of the paper, i.e., some general properties of (the complement of) the set of (strong) activity are addressed in Section III. Finally, conclusions are stated in Section IV.

\section{PRoblem STATEMENT AND PRELIMINARIES}

Consider the linear time-invariant system

$$
x(k+1)=A x(k)+B u(k), \quad x(0)=x_{0}
$$

with state and input constraints of the form

$$
x(k) \in \mathcal{X} \subset \mathbb{R}^{n} \text { and } u(k) \in \mathcal{U} \subset \mathbb{R}^{m} \text { for every } k \in \mathbb{N},
$$

where $\mathcal{X}$ and $\mathcal{U}$ are $\mathrm{C}$-sets (convex and compact sets that contain the origin as an interior point). The control task is to steer the system towards the origin while minimizing a

M. Schulze Darup and M. Cannon are with the Control Group, Department of Engineering Science, University of Oxford, UK. E-mail: moritz.schulzedarup@rub. de (quadratic) performance index. Taking the system dynamics and the constraints into account, this task can be formulated as the OCP

$$
\begin{array}{lll}
V_{N}^{\mathcal{C}}\left(x_{0}\right):=\min _{\mathbf{x}_{N}, \mathbf{u}_{N}}\|x(N)\|_{P}^{2}+\sum_{k=0}^{N-1}\|x(k)\|_{Q}^{2}+\|u(k)\|_{R}^{2} & \\
\text { s.t. } & x(0)=x_{0}, & \\
& x(k+1)=A x(k)+B u(k), & \forall k \in \mathbb{N}_{[0, N-1]}, \\
& x(k) \in \mathcal{X}, & \forall k \in \mathbb{N}_{[0, N-1]}, \\
& u(k) \in \mathcal{U}, & \\
& x(N) \in \mathcal{C}, &
\end{array}
$$

where $\|x\|_{P}^{2}:=x^{T} P x$ and $\mathbb{N}_{\mathcal{I}}:=\{k \in \mathbb{N} \mid k \in \mathcal{I}\}$. Moreover, $N \in \mathbb{N}_{[1, \infty)}$ is the prediction horizon, $P \in \mathbb{R}^{n \times n}$, $Q \in \mathbb{R}^{n \times n}$, and $R \in \mathbb{R}^{m \times m}$ are positive definite weighting matrices, and $\mathcal{C}$ is a $\mathrm{C}$-set, which operates as a terminal constraint. Finally, the decision variables are denoted by

$\mathbf{x}_{N}:=\left(\begin{array}{c}x(0) \\ \vdots \\ x(N)\end{array}\right) \in \mathbb{R}^{(N+1) n}$ and $\mathbf{u}_{N}:=\left(\begin{array}{c}u(0) \\ \vdots \\ u(N-1)\end{array}\right) \in \mathbb{R}^{N m}$.

The terminal weighting $P$ is chosen so that it solves the discrete-time algebraci Riccati equation (DARE)

$$
A^{T}\left(P-P B\left(R+B^{T} P B\right)^{-1} B^{T} P\right) A-P+Q=0
$$

associated with the unconstrained infinite horizon cost. The terminal set $\mathcal{C}$ is either chosen equal to the state constraint set, i.e., $\mathcal{C}=\mathcal{X}$ or as a suitable terminal set $\mathcal{T}$, which satisfies the following conditions (cf. [5, Assum. A1-A3]). Note that [5, Assum. A4] holds by construction, since $\|x\|_{P}^{2}$ is a local Lyapunov function.

Condition 1: Let $P \succ 0$ solve (3) and let

$$
K:=-\left(R+B^{T} P B\right)^{-1} B^{T} P A .
$$

We call a C-set $\mathcal{T} \subset \mathbb{R}^{n}$ a suitable terminal set, if

(i) $\mathcal{T} \subseteq \mathcal{X}$ (state constraint satisfaction),

(ii) $K x \in \mathcal{U}$ for every $x \in \mathcal{T}$ (input constraint satisfaction),

(iii) $(A+B K) x \in \mathcal{T}$ for every $x \in \mathcal{T}$ (positive invariance).

The largest set $\mathcal{T}^{*}$ satisfying Cond. 1 can be described by

$$
\mathcal{T}^{*}:=\left\{x_{0} \in \mathcal{D} \mid(A+B K)^{k} x_{0} \in \mathcal{D}, \forall k \in \mathbb{N}_{[1, M]}\right\},
$$

where $\mathcal{D}:=\{x \in \mathcal{X} \mid K x \in \mathcal{U}\}$ and where $M \in \mathbb{N}$ is a sufficiently large number (see [7] for details). Now, based on (2), we define the MPC scheme

$$
\varrho_{N}^{\mathcal{C}}\left(x_{0}\right):=u^{*}(0)
$$

where $u^{*}(0)$ is the first input of the optimal input sequence $\mathbf{u}_{N}^{*}$. For the choice $\mathcal{C}=\mathcal{T}$, it is well-known (see, e.g., [5]) that the control scheme (6) guarantees exponential stability of the controlled system $x(k+1)=A x(k)+B \varrho_{N}^{\mathcal{C}}(x(k))$. 
However, depending on the system, the constraints, and the horizon length, the choice $\mathcal{C}=\mathcal{T}$ may result in a significantly smaller feasible set

$$
\mathcal{F}_{N}^{\mathcal{C}}:=\left\{x_{0} \in \mathcal{X} \mid(2) \text { is feasible }\right\}
$$

than the choice $\mathcal{C}=\mathcal{X}$. Moreover, the choice $\mathcal{C}=\mathcal{T}$ may force trajectories of the controlled system to enter $\mathcal{T}$ earlier than the optimal trajectory (achieved for $N \rightarrow \infty$ ) would do. In this context, we define the set of (strong) activity ${ }^{1}$

$$
\mathcal{S}_{N}:=\left\{x_{0} \in \mathcal{F}_{N}^{\mathcal{T}} \mid V_{N}^{\mathcal{T}}\left(x_{0}\right)>V_{N}^{\mathcal{X}}\left(x_{0}\right)\right\}
$$

which collects all initial states $x_{0}$ for which the terminal constraint $\mathcal{C}=\mathcal{T}$ is strongly active, thus increasing the performance index. In other words, $\mathcal{S}_{N}$ contains all initial conditions for which the solution of (2) differs from the solution of the CLQR problem (see [2] for details). In addition, we define the complement

$$
\mathcal{S}_{N}^{c}:=\mathcal{F}_{N}^{\mathcal{T}} \backslash \mathcal{S}_{N}
$$

which collects all initial states $x_{0}$ for which the terminal constraint is weakly active or inactive. Since $V_{N}^{\mathcal{T}}\left(x_{0}\right) \geq$ $V_{N}^{\mathcal{X}}\left(x_{0}\right)$ for every $x_{0} \in \mathcal{F}_{N}^{\mathcal{T}}$ by construction, $\mathcal{S}_{N}^{c}$ can also be written as $\mathcal{S}_{N}^{c}=\left\{x_{0} \in \mathcal{F}_{N}^{\mathcal{T}} \mid V_{N}^{\mathcal{T}}\left(x_{0}\right)=V_{N}^{\mathcal{X}}\left(x_{0}\right)\right\}$. Although the OCP (2) and the associated controller (6) are only meaningful for $N \in \mathbb{N}_{[1, \infty)}$, the analysis in this paper is facilitated by formally defining $V_{0}^{\mathcal{C}}\left(x_{0}\right):=\left\|x_{0}\right\|_{P}^{2}$ and $\mathcal{F}_{0}^{\mathcal{C}}:=\mathcal{C}$. Inspired by (8) and (9), this gives rise to $\mathcal{S}_{0}:=\emptyset$ and $\mathcal{S}_{0}^{c}:=\mathcal{T}$. In the following, we study properties of the set $\mathcal{S}_{N}^{c}$ (resp. $\mathcal{S}_{N}$ ). In particular, we are interested in the relation between the sets $\mathcal{S}_{N}^{c}$ and $\mathcal{F}_{N}^{\mathcal{T}}$ for different $N \in \mathbb{N}$.

\section{PROPERTIES OF THE SET OF ACTIVITY}

\section{A. The complement is compact and nested}

It is well-known that the sets $\mathcal{F}_{N}^{\mathcal{T}}$ and $\mathcal{F}_{N}^{\mathcal{X}}$ are C-sets for every $N \in \mathbb{N}$ (given $\mathcal{T}, \mathcal{X}$, and $\mathcal{U}$ are C-sets). Moreover, we have $\mathcal{F}_{N}^{\mathcal{T}} \subseteq \mathcal{F}_{N+1}^{\mathcal{T}} \subseteq \mathcal{F}_{N+1}^{\mathcal{X}} \subseteq \mathcal{F}_{N}^{\mathcal{X}}$ since $\mathcal{T}$ is invariant, due to $\mathcal{T} \subseteq \mathcal{X}$, and by definition of the state constraints in (2), respectively. Similar properties hold for the complement of the set of activity as summarized in Thms. 1 and 2. As a preparation, note that we have

$$
V_{N}^{\mathcal{X}}\left(x_{0}\right) \leq V_{N+1}^{\mathcal{X}}\left(x_{0}\right) \leq V_{N+1}^{\mathcal{T}}\left(x_{0}\right) \leq V_{N}^{\mathcal{T}}\left(x_{0}\right)
$$

for every $N \in \mathbb{N}$ and every $x_{0} \in \mathcal{F}_{N}^{\mathcal{T}}$ by construction.

Theorem 1: We have $\mathcal{S}_{N}^{c} \subseteq \mathcal{S}_{N+1}^{c}$ for every $N \in \mathbb{N}$.

Proof: Consider any $N \in \mathbb{N}$ and assume $\mathcal{S}_{N}^{c} \nsubseteq \mathcal{S}_{N+1}^{c}$. Then there exists an $x_{0} \in \mathcal{S}_{N}^{c}$ such that $x_{0} \notin \mathcal{S}_{N+1}^{c}$. Having $x_{0} \notin \mathcal{S}_{N+1}^{c}$ implies $V_{N+1}^{\mathcal{T}}\left(x_{0}\right)>V_{N+1}^{\mathcal{X}}\left(x_{0}\right)$. We thus obtain $V_{N}^{\mathcal{T}}\left(x_{0}\right)>V_{N}^{\mathcal{X}}\left(x_{0}\right)$ according to (10). This, however, contradicts $x_{0} \in \mathcal{S}_{N}^{c}$ which implies $V_{N}^{\mathcal{T}}\left(x_{0}\right)=V_{N}^{\mathcal{X}}\left(x_{0}\right)$.

Theorem 2: For every $N \in \mathbb{N}$, the set $\mathcal{S}_{N}^{c}$ is compact and contains the origin as an interior point.

Proof: The claim holds by definition for $N=0$ since $\mathcal{T}$ is a C-set. For every $N \in \mathbb{N}_{[1, \infty)}$, we have $\mathcal{S}_{N}^{c} \subseteq \mathcal{F}_{N}^{\mathcal{T}} \subseteq \mathcal{X}$ by construction and $\mathcal{T} \subseteq \mathcal{S}_{N}^{c}$ according to Thm. 1. Thus, $\mathcal{S}_{N}^{c}$ is bounded and contains the origin as an interior point. It remains to show that $\mathcal{S}_{N}^{c}$ is closed. By definition, we have $V_{N}^{\mathcal{T}}\left(x_{0}\right)=V_{N}^{\mathcal{X}}\left(x_{0}\right)$ for every $x_{0} \in \mathcal{S}_{N}^{c}$. Thus, $\mathcal{S}_{N}^{c}$ not being

\footnotetext{
${ }^{1}$ Regions of activity for constraints in MPC were first introduced in [8]. There, however, the focus was not on terminal constraints.
}

closed contradicts continuity of $V_{N}^{\mathcal{T}}(\cdot)$ and $V_{N}^{\mathcal{X}}(\cdot)$, which is given according to [1, Lem. 4$]$.

Clearly, Thm. 1 implies $x_{0} \in \mathcal{S}_{k}^{c}$ for every $k \geq N$ whenever $x_{0} \in \mathcal{S}_{N}^{c}$. Consequently, we have

$$
V_{N}^{\mathcal{T}}\left(x_{0}\right)=V_{k}^{\mathcal{T}}\left(x_{0}\right)=V_{k}^{\mathcal{X}}\left(x_{0}\right)=V_{\infty}^{\mathcal{X}}\left(x_{0}\right)
$$

for every $k \in \mathbb{N}_{[N, \infty)}$ and $x_{0} \in \mathcal{S}_{N}^{c}$, where $V_{\infty}^{\mathcal{X}}\left(x_{0}\right)$ is, by definition, the performance index of the CLQR (resulting from (2) for $N \rightarrow \infty$ and $\mathcal{C}=\mathcal{X}$ ). In other words, $\mathcal{S}_{N}^{c}$ contains all states for which the solution of (2) coincides with the solution of the CLQR problem (for the first $N$ steps).

\section{B. The complement may or may not be convex}

In the previous section, we recalled that $\mathcal{F}_{N}^{\mathcal{T}}$ and $\mathcal{F}_{N}^{\mathcal{X}}$ are $\mathrm{C}$-sets and showed that $\mathcal{S}_{N}^{c}$ is compact and contains the origin as an interior point. Clearly, it remains to study whether $\mathcal{S}_{N}^{c}$ is convex or not, i.e., whether $\operatorname{conv}\left(\mathcal{S}_{N}^{c}\right)=\mathcal{S}_{N}^{c}$ holds (where $\operatorname{conv}\left(\mathcal{S}_{N}^{c}\right)$ denotes the convex hull of the set $\left.\mathcal{S}_{N}^{c}\right)$. In this context, we obviously have $\operatorname{conv}\left(\mathcal{S}_{N}^{c}\right) \subseteq \mathcal{F}_{N}^{\mathcal{T}}$ due to $\mathcal{S}_{N}^{c} \subseteq \mathcal{F}_{N}^{\mathcal{T}}$ and since $\mathcal{F}_{N}^{\mathcal{T}}$ is convex. At first sight, it may appear that $\mathcal{S}_{N}^{c}$ should be convex for any $N$. In fact, for every $x_{0} \in \operatorname{conv}\left(\mathcal{S}_{N}^{c}\right)$, there exist some $x_{1}, \ldots, x_{M} \in \mathcal{S}_{N}^{c}$ and $\alpha_{1}, \ldots, \alpha_{M} \in[0,1]$ such that $x_{0}=\sum_{i=1}^{M} \alpha_{i} x_{i}$ and $\sum_{i=1}^{M} \alpha_{i}=1$. We have $V_{N}^{\mathcal{T}}\left(x_{i}\right)=V_{N}^{\mathcal{X}}\left(x_{i}\right)$ for every $i \in \mathbb{N}_{[1, M]}$ by construction. In other words, for every $x_{i}$, the terminal constraints are not strongly active. Clearly, by interpolating the optimal state and input sequences associated with the states $x_{i}$, we can construct a feasible solution for (2) with $\mathcal{C}=\mathcal{T}$, for which the terminal constraints are likewise not strongly active. The existence of such a feasible solution does, however, not imply that the optimal solution comes with inactive (or weakly active) terminal constraints as the following example illustrates.

Example 1: Consider the double integrator in [9] with

$$
A=\left(\begin{array}{ll}
1 & 1 \\
0 & 1
\end{array}\right) \quad \text { and } \quad B=\left(\begin{array}{c}
0.5 \\
1
\end{array}\right)
$$

and the constraints $\mathcal{X}=\left\{x \in \mathbb{R}^{2}|| x_{1}|\leq 25,| x_{2} \mid \leq 5\right\}$ and $\mathcal{U}=[-1,1]$. Choosing the weighting matrices $Q=I_{2}$ and $R=0.1$ implies

$P=\left(\begin{array}{ll}2.0599 & 0.5916 \\ 0.5916 & 1.4228\end{array}\right)$ and $K=\left(\begin{array}{ll}-0.6167 & -1.2703\end{array}\right)$ according to (3) and (4). Computing the largest terminal set as in (5) (with $M=2$ ) results in

$\mathcal{T}=\left\{x_{0} \in \mathbb{R}^{2} \mid \pm W x_{0} \leq \mathbf{1}\right\}$ with $W=\left(\begin{array}{ll}0.6167 & 1.2703 \\ 0.3569 & 0.1184\end{array}\right)$.

Now, consider $N=1$ and note that $\mathcal{S}_{1}^{c}$ is not convex (see Fig. 1). In fact, the initial state $x_{0}=\left(\begin{array}{lll}-4.00 & 1.16\end{array}\right)^{T}$ satisfies $x_{0} \in \operatorname{conv}\left(\mathcal{S}_{1}^{c}\right)$ but $x_{0} \notin \mathcal{S}_{1}^{c}$. However, $x_{0}$ can be written as the convex combination $x_{0}=\alpha_{1} \xi_{1}+\alpha_{2} \xi_{2}$ with

$$
\xi_{1}=\left(\begin{array}{ll}
-4.6 & 1.0
\end{array}\right)^{T} \in \mathcal{S}_{1}^{c} \text { and } \xi_{2}=\left(\begin{array}{ll}
-1.0 & 1.8
\end{array}\right)^{T} \in \mathcal{S}_{1}^{c}
$$

and $\alpha_{1}=1-\alpha_{2}=0.8$. Evaluating the MPC law (6) with $\mathcal{C}=\mathcal{X}$ for the three initial conditions yields

$$
\varrho_{1}^{\mathcal{X}}\left(x_{0}\right)=0.9932, \quad \varrho_{1}^{\mathcal{X}}\left(\xi_{1}\right)=1.0, \text { and } \varrho_{1}^{\mathcal{X}}\left(\xi_{2}\right)=-1.0 \text {. }
$$

As presupposed by $\xi_{1}, \xi_{2} \in \mathcal{S}_{1}^{c}$, the successors of $\xi_{1}$ and $\xi_{2}$ are contained in $\mathcal{T}$ (see Fig. 1). Moreover, the interpolated 
input $\hat{u}_{0}:=\alpha_{1} \varrho_{1}^{\mathcal{X}}\left(\xi_{1}\right)+\alpha_{2} \varrho_{1}^{\mathcal{X}}\left(\xi_{2}\right)=0.6 \in \mathcal{U}$ is such that $\hat{x}_{0}^{+}:=A x_{0}+B \hat{u}_{0} \in \mathcal{T}$. In contrast, as illustrated in Fig. 1, the successor of $x_{0}$ for the optimal input $\varrho_{1}^{\mathcal{X}}\left(x_{0}\right)$ is not contained in $\mathcal{T}$ (and not even in $\mathcal{F}_{1}^{\mathcal{T}}$ ). Roughly speaking, this behavior can be observed since the terminal set $\mathcal{T}$ is not a level set of the terminal cost $\|x\|_{P}^{2}$ and since $\|x\|_{P}^{2}$ only provides an underestimator of the infinite horizon cost $V_{\infty}^{\mathcal{X}}(x)$ for every $x \notin \mathcal{T}$. (a) $N=1$

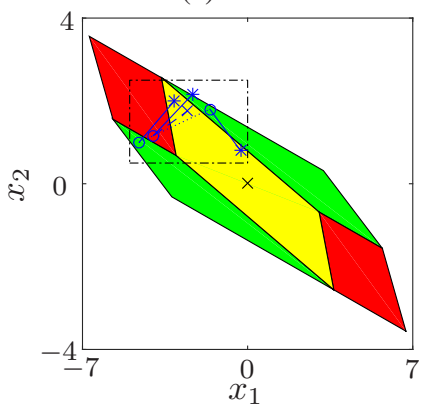

(b) detail

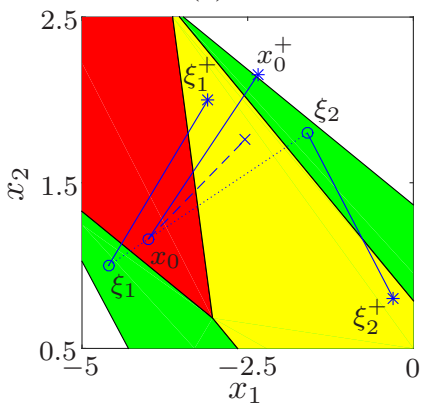

Fig. 1. Illustration of (the complement of) the set of activity for the system in Exmp. 1 for $N=1$. In (a), the yellow set shows the terminal set $\mathcal{T}$. The union of the yellow and the green sets is $\mathcal{S}_{1}^{c}$ and the union of the red sets is $\mathcal{S}_{1}$. The dash-dotted rectangle marks the detail in (b). The blue solid lines illustrate the optimal solution for (2) with $\mathcal{C}=\mathcal{T}$ and $N=1$ for the initial conditions $\xi_{1}, \xi_{2}$, and $x_{0}$ (marked by circles). The successors are labeled with $\xi_{1}^{+}, \xi_{2}^{+}$, and $x_{0}^{+}$, respectively. For the initial state $x_{0} \in \mathcal{S}_{1}$, the dashed line depicts a non-optimal trajectory terminating at $\hat{x}_{0}^{+} \in \mathcal{T}$ (marked with a blue cross). This trajectory was obtained by interpolating the optimal trajectories for the states $\xi_{1}, \xi_{2} \in \mathcal{S}_{1}^{c}$.

The example illustrates that $\mathcal{S}_{N}^{c}$ may be non-convex. In fact, for the discussed example, $\mathcal{S}_{N}^{c}$ is non-convex for every $N \in \mathbb{N}_{[1,13]}$ (see Fig. 1, Fig. 2.(a), and Fig. 2.(b) for the cases $N \in\{1,12,13\})$. However, $\mathcal{S}_{N}^{c}$ may also be convex. In fact, for the same example, $\mathcal{S}_{N}^{c}$ is convex for every $N \geq 14$ (see Fig. 2.(c), and Fig. 2.(d) for the cases $N \in\{14,15\}$ ). Thus, in contrast to the convex sets $\mathcal{F}_{N}^{\mathcal{T}}$ and $\mathcal{F}_{N}^{\mathcal{X}}, \mathcal{S}_{N}^{c}$ may or may not be convex (i.e., $\mathcal{S}_{N}^{c}$ may or may not be a C-set).

\section{The set of activity may or may not be empty}

Studying the set of activity $\mathcal{S}_{N}$ for the system in Exmp. 1 and different $N \in \mathbb{N}_{[1, \infty)}$, we make an interesting observation. For $N=15$, we have $\mathcal{S}_{N}^{c}=\mathcal{F}_{N}^{\mathcal{T}}$ and consequently $\mathcal{S}_{N}=\emptyset$. This observation raises the question whether there always exists a finite $N \in \mathbb{N}_{[1, \infty)}$ such that $\mathcal{S}_{N}$ is empty. It is important to note that a very similar question has already been addressed in a different context. In fact, stabilizing MPC schemes without terminal constraints implicitly require a choice of $N$ such that potential terminal constraints would not be strongly active for a certain set of initial conditions (see the proofs of [6, Thm. 12 and Thm. 13]). Rewriting and condensing the statements in [6, Thm. 12 and Thm. 13] using the notation introduced in this paper results in the following theorem, where the $\mathrm{C}$-set $\mathcal{F}_{\infty}^{\mathcal{X}}$ is the maximal controlled invariant set.

Theorem 3: For every $\lambda \in(0,1)$, there exists a finite $N \in$ $\mathbb{N}$ such that

$$
\lambda \mathcal{F}_{\infty}^{\mathcal{X}} \subseteq \mathcal{S}_{k}^{c} \quad \text { for every } \quad k \geq N
$$

In principle, the proof of Thm. 3 can be easily derived from the proofs of [6, Thm. 12 and Thm. 13]. Nevertheless, we provide a self-contained proof for Thm. 3 in App. C for two reasons. First, we reuse the ideas in the proof of Thm. 3 to prove Thm. 4 further below. Second, the proof of Thm. 3 comes with an overestimation of a suitable horizon length $N$. We were able to significantly improve this overestimation compared to the original proof in [6] (see Rem. 1 in the appendix for details). This byproduct may be useful for stabilizing MPC schemes without terminal constraints.

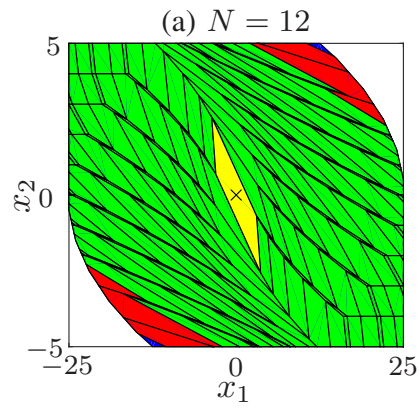

(c) $N=14$

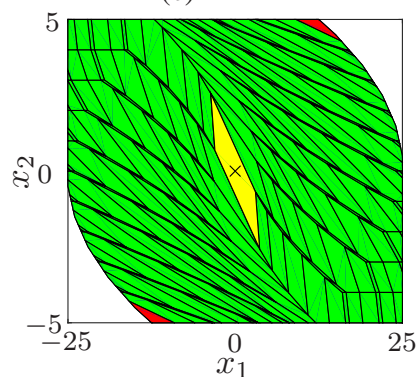

(b) $N=13$

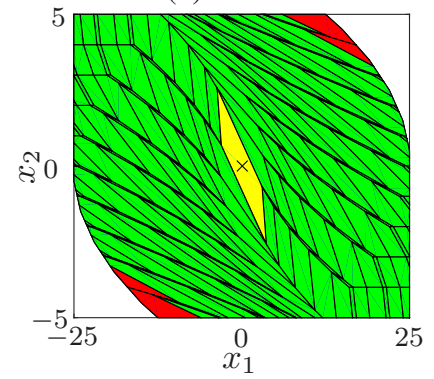

(d) $N=15$

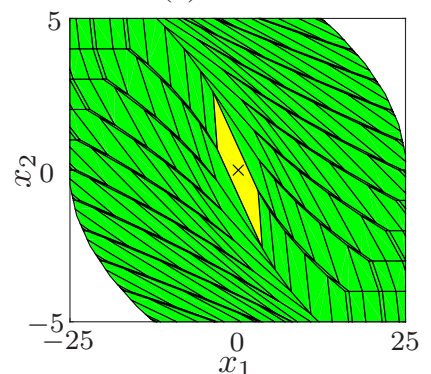

Fig. 2. Illustration of (the complement of) the set of activity for the system in Exmp. 1 for different prediction horizons $N$. The yellow set shows the terminal set $\mathcal{T}$. The union of the yellow and the green sets refers to $\mathcal{S}_{N}^{c}$ and the union of the red sets depicts $\mathcal{S}_{N}$. The union of the yellow, the green, the red and the blue (only visible in (a)) sets illustrates the maximal controlled invariant set $\mathcal{F}_{\infty}^{\mathcal{X}}$.

Now, since we have $\mathcal{F}_{N}^{\mathcal{T}} \subseteq \mathcal{F}_{\infty}^{\mathcal{X}}$ by construction, Thm. 3 immediately implies that, for every $\lambda \in(0,1)$, there exists $N \in \mathbb{N}$ such that

$$
\lambda \mathcal{F}_{N}^{\mathcal{T}} \subseteq \mathcal{S}_{k}^{c} \quad \text { for every } \quad k \geq N
$$

Clearly, if (12) would also hold for $\lambda=1$ (and some $N \in \mathbb{N}$ ), the set of activity would always be empty for a finite $N$. To see this, note that (12) with $\lambda=1$ in combination with (9) implies $\mathcal{F}_{N}^{\mathcal{T}} \subseteq \mathcal{S}_{N}^{c} \subseteq \mathcal{F}_{N}^{\mathcal{T}}$, i.e., $\mathcal{S}_{N}^{c}=\mathcal{F}_{N}^{\mathcal{T}}$ and consequently $\mathcal{S}_{N}=\emptyset$. Unfortunately, it is easy to show that Thm. 3 cannot be extended to the special case $\lambda=1$. In fact, it is wellknown that there may exist initial states on the boundary of $\mathcal{F}_{\infty}^{\mathcal{X}}$ that cannot be steered towards the origin (see, e.g., $x_{0}=$ $5 \in \partial \mathcal{F}_{\infty}^{\mathcal{X}}$ for the system in Exmp. 2 below). Obviously, for such initial states $x_{0}$, the OCP (2) with $\mathcal{C}=\mathcal{T}$ is infeasible for every $N \in \mathbb{N}$, i.e., $x_{0} \notin \mathcal{F}_{N}^{\mathcal{T}} \supseteq \mathcal{S}_{N}^{c}$. This argument, however, does not apply to the weaker condition (12). Indeed, every $x_{0} \in \mathcal{F}_{N}^{\mathcal{T}}$ can be steered to $\mathcal{T}$ in at most $N$ steps by construction. Due to this circumstance, it is possible to guarantee $\mathcal{F}_{N}^{\mathcal{T}}=\mathcal{S}_{N}^{c}$ at least for a special class of systems including the system in Exmp. 1. In fact, as summarized in Thm. $4, \mathcal{F}_{N}^{\mathcal{T}}=\mathcal{S}_{N}^{c}$ can always be guaranteed if $\mathcal{F}_{\infty}^{\mathcal{X}}$ is finitely determined, i.e., if

$$
\mathcal{F}_{M}^{\mathcal{T}}=\mathcal{F}_{k}^{\mathcal{T}}=\mathcal{F}_{\infty}^{\mathcal{X}} \quad \text { for every } \quad k \geq M
$$

for some $M \in \mathbb{N}$. 
Theorem 4: Assume (13) holds for some $M \in \mathbb{N}$. Then there exists a finite $N \in \mathbb{N}$ such that $\mathcal{S}_{N}=\emptyset$.

Proof: Let $\bar{p}, \bar{q}, \bar{r}, \underline{p}, \underline{q} \in \mathbb{R}_{+}$be such that

$$
\begin{aligned}
& \bar{p} \geq \max _{x \in \mathcal{T}}\|x\|_{P}^{2}, \quad \bar{q} \geq \max _{x \in \mathcal{F}}\|x\|_{Q}^{2}, \quad \bar{r} \geq \max _{u \in \mathcal{U}}\|u\|_{R}^{2} \\
& \underline{p} \leq \inf _{x \in \mathcal{F}_{\infty}^{\mathcal{X}} \backslash \mathcal{T}}\|x\|_{P}^{2}, \quad \text { and } \quad \underline{q} \leq \inf _{x \in \mathcal{F}_{\infty}^{\mathcal{X}} \backslash \mathcal{T}}\|x\|_{Q}^{2},
\end{aligned}
$$

where $\mathbb{R}_{+}$denotes positive reals and choose $N \in \mathbb{N}$ such that

$$
N \geq \frac{1}{q}(\bar{p}-\underline{p}+(M-1) \bar{q}+M \bar{r})+1
$$

Assume $\mathcal{S}_{N} \neq \emptyset$. Then there exists an $x_{0} \in \mathcal{S}_{N}$ such that $V_{N}^{\mathcal{T}}\left(x_{0}\right)>V_{N}^{\mathcal{X}}\left(x_{0}\right)$. By construction, we have $\bar{p} \geq \underline{p}$ and $\bar{q} \geq$ $\underline{q}$ and thus $N \geq M$. Hence, we find $\mathcal{F}_{N}^{\mathcal{T}}=\mathcal{F}_{M}^{\mathcal{T}}$ according to (13) and consequently $x_{0} \in \mathcal{F}_{M}^{T}$. Moreover, corresponding to (10), we obtain $V_{N}^{\mathcal{T}}\left(x_{0}\right) \leq V_{M}^{\mathcal{T}}\left(x_{0}\right)$. Now, using the bounds from (14), $V_{M}^{\mathcal{T}}\left(x_{0}\right)$ can be overestimated as

$$
V_{M}^{\mathcal{T}}\left(x_{0}\right) \leq \bar{p}+\left\|x_{0}\right\|_{Q}^{2}+(M-1) \bar{q}+M \bar{r} .
$$

On the other hand, $V_{N}^{\mathcal{X}}\left(x_{0}\right)$ can be underestimated by

$$
V_{N}^{\mathcal{X}}\left(x_{0}\right) \geq \underline{p}+\left\|x_{0}\right\|_{Q}^{2}+(N-1) \underline{q} .
$$

To see this, note that the the optimizers $\mathbf{x}_{N}^{*}$ and $\mathbf{u}_{N}^{*}$ for (2) with $\mathcal{C}=\mathcal{X}$ satisfy $x^{*}(N) \notin \mathcal{T}$ due to $V_{N}^{\mathcal{T}}\left(x_{0}\right)>V_{N}^{\mathcal{X}}\left(x_{0}\right)$. Clearly, $x^{*}(N) \notin \mathcal{T}$ immediately implies $x^{*}(k) \notin \mathcal{T}$ for every $k \in \mathbb{N}_{[0, N-1]}$ and thus $\left\|x^{*}(k)\right\|_{Q}^{2} \geq \underline{q}$. Taking $\left\|u^{*}(k)\right\|_{R}^{2} \geq 0$ into account yields (17). Combining the overestimation for $V_{N}^{\mathcal{T}}\left(x_{0}\right)$ and the underestimation for $V_{N}^{\mathcal{X}}\left(x_{0}\right)$ shows that $V_{N}^{\mathcal{T}}\left(x_{0}\right)>V_{N}^{\mathcal{X}}\left(x_{0}\right)$ can only be true if

$$
\bar{p}+(M-1) \bar{q}+M \bar{r}>\underline{p}+(N-1) \underline{q} .
$$

This, however, contradicts (15).

A necessary and sufficient condition for (13) to hold is

$$
\mathcal{F}_{M}^{\mathcal{T}}=\mathcal{F}_{M+1}^{\mathcal{T}}
$$

for some $M \in \mathbb{N}$ (cf. [10, Thm. 2.2]). For the system in Exmp. 1, (18) is given for $M=13$ (see Fig. 2). One could think that (18) is necessary (and sufficient) for observing $\mathcal{S}_{N}=\emptyset$ for some $N \geq M$. This is, however, not true as the following example shows.

Example 2: Consider system (1) with the matrices $A=\frac{6}{5}$ and $B=1$ and the constraints $\mathcal{X}=[-10,10]$ and $\mathcal{U}=$ $[-1,1]$. Let $Q=\frac{19}{5}$ and $R=1$. Then, $P=5$ solves (3) and $K=-1$ according to (4). Moreover, we obtain $\mathcal{D}=[-1,1]$ and consequently the (largest) terminal set $\mathcal{T}=\mathcal{D}=[-1,1]$ according to (5) (with $M=0$ ). In addition, it is easy to see that we have

$$
\mathcal{F}_{k}^{\mathcal{T}}=\left[-f_{k}^{\mathcal{T}}, f_{k}^{\mathcal{T}}\right], \quad \mathcal{F}_{\infty}^{\mathcal{X}}=[-5,5], \quad \text { and } \mathcal{F}_{k}^{c}=\left[-f_{k}^{\mathcal{X}}, f_{k}^{\mathcal{X}}\right]
$$

for every $k \in \mathbb{N}$, where $f_{k}^{\mathcal{T}}$ and $f_{k}^{\mathcal{X}}$ are defined via $f_{k}^{\mathcal{C}}:=$ $\frac{5}{6}\left(f_{k-1}^{\mathcal{C}}+1\right)$ with $\mathcal{C}=\mathcal{T}$ or $\mathcal{C}=\mathcal{X}, f_{0}^{\mathcal{T}}:=1$, and $f_{0}^{\mathcal{X}}:=$ 10. We obviously find $f_{M}^{\mathcal{T}}<f_{M+1}^{\mathcal{T}}<5$ and thus $\mathcal{F}_{M}^{\mathcal{T}} \subset$ $\mathcal{F}_{M+1}^{\mathcal{T}} \subset \mathcal{F}_{\infty}^{\mathcal{X}}$ for every $M \in \mathbb{N}$. In the following, let $N=1$, note that $f_{1}^{\mathcal{T}}=\frac{5}{3}$ and $f_{1}^{\mathcal{X}}=\frac{55}{6}$, and consider any $x_{0} \in \mathcal{F}_{1}^{\mathcal{X}}$. In this case, the OCP (2) with $\mathcal{C}=\mathcal{X}$ simplifies to

$$
\begin{aligned}
& V_{1}^{\mathcal{X}}\left(x_{0}\right)=\min _{u_{0}} 6 u_{0}^{2}+12 u_{0} x_{0}+11 x_{0}^{2} \\
& \text { s.t. } \pm u_{0}-1 \leq 0 \text { and } \pm\left(6 x_{0}+5 u_{0}\right)-50 \leq 0 .
\end{aligned}
$$

By analyzing the Karush-Kuhn-Tucker (KKT) conditions, we identify $u_{0}^{*}=-\operatorname{sign}\left(x_{0}\right) \min \left\{1,\left|x_{0}\right|\right\}$ to be the optimizer for (2) and $x_{0} \in \mathcal{F}_{1}^{\mathcal{X}}$ (see App. A for details). This makes sense, since we recover the LQR law $u_{0}^{*}=-K x_{0}=-x_{0}$ for every $x_{0} \in \mathcal{T}$. In addition, it is easy to check that we have $A x_{0}+B u_{0}^{*} \in \mathcal{T}$ for every $x_{0} \in \mathcal{F}_{1}^{\mathcal{T}}$. In other words, the terminal constraints (i.e., $x(1) \in \mathcal{T}$ ) will not be strongly active while solving OCP (2) with $\mathcal{C}=\mathcal{T}$ for any $x_{0} \in \mathcal{F}_{1}^{\mathcal{T}}$ and thus $\mathcal{F}_{1}^{\mathcal{T}}=\mathcal{S}_{1}^{c}$. Along the same line of argumentation, it can be shown that $\mathcal{F}_{N}^{\mathcal{T}}=\mathcal{S}_{N}^{c}$ for every $N \in \mathbb{N}$.

Example 2 suggests that an $N$ such that (12) holds for $\lambda=1$ may exist even for systems which do not satisfy (13). In general, this is however not true as the following counterexample shows.

Example 3: Consider system (1) with the matrices

$$
A=\left(\begin{array}{ll}
0 & 1 \\
1 & 0
\end{array}\right) \quad \text { and } \quad B=\left(\begin{array}{l}
2 \\
4
\end{array}\right)
$$

and the constraints $\mathcal{X}=\left\{\left.x \in \mathbb{R}^{2}|| x\right|_{\infty} \leq 5\right\}$ and $\mathcal{U}=$ $[-1,1]$. Choosing $Q=I_{2}$ and $R=\frac{9}{2}$ results in

$$
P=\left(\begin{array}{rr}
\frac{5}{3} & -\frac{5}{6} \\
-\frac{5}{6} & \frac{8}{3}
\end{array}\right) \quad \text { and } K=\left(\begin{array}{ll}
-\frac{2}{9} & 0
\end{array}\right) .
$$

Computing the terminal set as in (5) (with $M=2$ ) yields

$$
\mathcal{T}=\left\{x_{0} \in \mathbb{R}^{2} \mid \pm W x_{0} \leq \mathbf{1}\right\} \text { with } W=\left(\begin{array}{rr}
0 & \frac{1}{5} \\
\frac{2}{9} & 0 \\
\frac{8}{81} & -\frac{2}{9}
\end{array}\right) \text {. }
$$

We claim that the feasible set $\mathcal{F}_{N}^{\mathcal{T}}$ can be described by

$$
\mathcal{F}_{N}^{\mathcal{T}}=\operatorname{conv}\left\{ \pm\left(\begin{array}{l}
5 \\
5
\end{array}\right), \pm\left(\begin{array}{c}
w_{N-1} \\
-5
\end{array}\right), \pm\left(\begin{array}{c}
-5 \\
w_{N}
\end{array}\right)\right\}
$$

for every $N \in \mathbb{N}_{[1, \infty)}$, where $w_{k}:=5-\frac{7}{2^{k+1}}$ for every $k \in \mathbb{N}$. The sets $\mathcal{F}_{1}^{\mathcal{T}}$ and $\mathcal{F}_{2}^{\mathcal{T}}$ are illustrated in Fig. 3. Note that it is straightforward to prove (20). A formal proof is, however, not required for the following line of argumentation. Equation (20) suggests that $\mathcal{F}_{M}^{\mathcal{T}} \subset \mathcal{F}_{M+1}^{\mathcal{T}} \subset$ $\mathcal{F}_{\infty}^{\mathcal{X}}$ for every $M \in \mathbb{N}$ (which is indeed the case). In contrast, since $\mathcal{X}$ is controlled invariant $\left(u_{0}=0 \in \mathcal{U}\right.$ is such that $A x_{0}+B u_{0} \in \mathcal{X}$ for every $x_{0} \in \mathcal{X}$ ), we have $\mathcal{F}_{k}^{\mathcal{X}}=\mathcal{X}=\mathcal{F}_{\infty}^{\mathcal{X}}$ for every $k \in \mathbb{N}$. (a) $N=1$

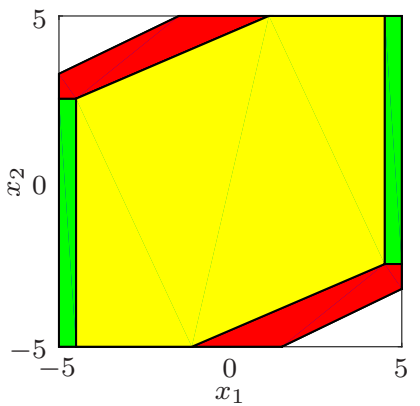

(b) $N=2$

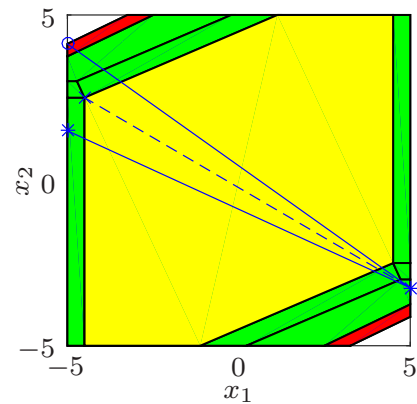

Fig. 3. Illustration of $\mathcal{S}_{N}$ (union of red sets) and $\mathcal{S}_{N}^{c}$ (union of yellow and green sets) for the system in Exmp. 3 and $N \in\{1,2\}$. The yellow set refers to the terminal set $\mathcal{T}$. For $N=2$, the solid and dashed trajectories depict the optimal solution for $(2)$ at $x_{0}=\left(\begin{array}{ll}-5 & w_{2}\end{array}\right)^{T}=\left(\begin{array}{ll}-5 & \frac{33}{8}\end{array}\right)^{T}$ with $\mathcal{C}=\mathcal{X}$ and $\mathcal{C}=\mathcal{T}$, respectively. 
In the following, we show that the initial state $x_{0}=$ $\left(\begin{array}{ll}-5 & w_{N}\end{array}\right)^{T}\left(\right.$ which is a vertex of $\left.\mathcal{F}_{N}^{\mathcal{T}}\right)$ satisfies $x_{0} \in \mathcal{S}_{N}$ for every $N \in \mathbb{N}_{[1, \infty)}$. To see this, first note that the input sequence $\mathbf{u}_{N}^{*}$ and the associated state sequence $\mathbf{x}_{N}^{*}$ with

$$
u^{*}(k):=\frac{(-1)^{k} \cdot 7}{2^{N+2-k}} \quad \text { and } \quad x^{*}(k):=(-1)^{k}\left(\begin{array}{c}
-5 \\
w_{N-k}
\end{array}\right)
$$

are optimizers for (2) with $\mathcal{C}=\mathcal{X}$ (see App. B for details). The sequences $\mathbf{u}_{N}^{*}$ and $\mathbf{x}_{N}^{*}$ are, however, not feasible for (2) with $\mathcal{C}=\mathcal{T}$ since

$$
x^{*}(N)=(-1)^{N}\left(\begin{array}{l}
-5 \\
w_{0}
\end{array}\right)=(-1)^{N}\left(\begin{array}{r}
-5 \\
\frac{3}{2}
\end{array}\right) \notin \mathcal{T} .
$$

Nevertheless, it can be shown that $x_{0} \in \mathcal{F}_{N}^{\mathcal{T}}$ (see App. B). Now, since (2) is a strictly convex OCP for $\mathcal{C}=\mathcal{X}$ and $\mathcal{C}=\mathcal{T}$, the observation that $\mathbf{u}_{N}^{*}$ and $\mathbf{x}_{N}^{*}$ are infeasible for (2) with $\mathcal{C}=\mathcal{T}$ but optimizers for (2) with $\mathcal{C}=\mathcal{X}$ implies $V_{N}^{\mathcal{T}}\left(x_{0}\right)>V_{N}^{\mathcal{X}}\left(x_{0}\right)$. Thus, $x_{0} \in \mathcal{S}_{N}$ and $\mathcal{S}_{N} \neq \emptyset$.

In summary, Exmps. 2 and 3 show that the set of activity may or may not be empty independent of the choice of prediction horizon $N$. In other words, an $N$ such that (12) holds for $\lambda=1$ will in general not exist.

\section{CONCLUSION}

We presented some observations on the activity of terminal constraints in stabilizing MPC for linear constrained systems. In particular, we proved that the complement of the set of activity (see Sect. II for a definition) is always compact (see Sect. III-A and Thm. 2) but it may or may not be convex (see Sect. III-B and Exmp. 1). Moreover, we showed that the set of activity may or may not be empty independent of the choice of the prediction horizon $N$, i.e., even for arbitrarily large $N$ (see Sect. III-C and Exmps. 2 and 3). An exception to this observation is the case where the largest controlled set is finitely determined (see Eq. 13). In fact, in this case, there always exists a finite $N$ such that the set of activity is empty (see Thm. 4).

The presented observations may be useful in the context of CLQR and stabilizing MPC without terminal constraints. For both applications, an algorithm searching for a horizon length $N$ such that the corresponding set of activity is empty would be desirable. The results in this paper show, however, that the successful termination of such an algorithms can in general not be guaranteed. Thus, future work has to address numerical methods to check (11) for a given $\lambda<1$ that do (in contrast to [3]) not require to solve (2) explicitly for all feasible initial states.

\section{ACKNOWLEDGMENT}

We thank Michael Jost (author of [8]) for fruitful discussions and support in an early stage of this work. We note that all figures were generated using the multi-parametric toolbox [11]. Finally, funding by the German Research Foundation (DFG) under the grants SCHU 2094/1-1 and SCHU 2094/2-1 is gratefully acknowledged.

\section{REFERENCES}

[1] D. Chmielewski and V. Manousiouthakis, "On constrained infinitetime linear quadratic optimal control," System and Control Letters, vol. 29, pp. 121-129, 1996.

[2] P. O. M. Scokaert and J. B. Rawlings, "Constrained linear quadratic regulation," IEEE Trans. Autom. Control, vol. 43, no. 8, pp. 11631169, 1998.

[3] P. Grieder, F. Borelli, F. Torrisi, and M. Morari, "Computation of the constrained infinite time linear quadratic regulator," in Proc. of 2003 American Control Conference, 2003, pp. 4711-4716.

[4] M. Sznaier and M. J. Damborg, "Suboptimal control of linear systems with state and control inequality constraints," in Proc. of 26 th Conference on Decision and Control, 1987, pp. 761-762.

[5] D. Q. Mayne, J. B. Rawlings, C. Rao, and P. O. M. Scokaert, "Constrained model predictive control: Stability and optimality," $\mathrm{Au}$ tomatica, vol. 36, pp. 789-814, 2000.

[6] A. Boccia, L. Grüne, and K. Worthmann, "Stability and feasibility of state constrained MPC without stabilizing terminal constraints," System and Control Letters, vol. 72, pp. 14-21, 2014.

[7] E. G. Gilbert and K. T. Tan, "Linear systems with state and control constraints: The theory and application of maximal output admissible sets," IEEE Trans. Autom. Control, vol. 36, pp. 1008-1020, 1991.

[8] M. Jost and M. Mönnigmann, "Accelerating online MPC with partial explicit information and linear storage complexity in the number of constraints," in Proc. of the 2013 European Control Conference, 2013, pp. $35-40$.

[9] P. O. Gutman and M. Cwikel, "An algorithm to find maximal state constraint sets for discrete-time linear dynamical systems with bounded control and states," IEEE Trans. Autom. Control, vol. 32, no. 3, pp. 251-253, 1987.

[10] E. C. Kerrigan, "Robust Constraint Satisfaction: Invariant Sets and Predictive Control," Ph.D. dissertation, University of Cambridge, 2000.

[11] M. Herceg, M. Kvasnica, C. N. Jones, and M. Morari, "MultiParametric Toolbox 3.0," in In Proc. of the 2013 European Control Conference, 2013, pp. 502-510.

[12] A. Bemporad, M. Morari, V. Dua, and E. N. Pistikopoulos, "The explicit linear quadratic regulator for constrained systems," Automatica, vol. 38, no. 1, pp. 3-20, 2002.

\section{APPENDIX}

\section{A. Details of Example 2}

According to the Karush-Kuhn-Tucker (KKT) conditions, $u_{0}^{*}$ is an optimizer for (19) if (and only if), in addition to primal feasibility, there exists $\lambda \in \mathbb{R}^{4}$ such that

$$
\begin{aligned}
12 x_{0}+12 u_{0}^{*}+\lambda_{1}-\lambda_{2}+\lambda_{3}-\lambda_{4} & =0, \\
\lambda_{1}\left(u_{0}^{*}-1\right) & =0, \\
\lambda_{2}\left(-u_{0}^{*}-1\right) & =0, \\
\lambda_{3}\left(1.2 x_{0}+u_{0}^{*}-10\right) & =0, \\
\lambda_{4}\left(-1.2 x_{0}-u_{0}^{*}-10\right) & =0,
\end{aligned}
$$

and $\lambda_{j} \geq 0$ for every $j \in \mathbb{N}_{[1,4]}$. It is easy to see that $u_{0}^{*}=-x_{0}$ is an optimizer for every $x_{0} \in[-1,1]$. In fact, we have primal feasibility since $u_{0}^{*} \in \mathcal{U}$ and $A x_{0}+B u_{0}^{*}=0.2 x_{0} \in \mathcal{X}$. In addition, the choice $\lambda_{i}=0$ solves (22) for every $x_{0} \in[-1,1]$. Now, consider any $x_{0} \in\left(1, \frac{55}{6}\right]$. Then, the optimal solution is $u_{0}^{*}=-1$, which can be easily proven using the Lagrange multipliers $\lambda_{1}=$ $\lambda_{3}=\lambda_{4}=0$ and $\lambda_{2}=12 x_{0}-12>0$. Analogously, we obtain $u_{0}^{*}=1$ and the Lagrange multipliers $\lambda_{2}=\lambda_{3}=\lambda_{4}=0$ and $\lambda_{1}=-12 x_{0}-12>0$ for every $x_{0} \in\left[-\frac{55}{6},-1\right)$.

\section{B. Details of Example 3}

To see that $\mathbf{u}_{N}^{*}$ and $\mathbf{x}_{N}^{*}$ are optimizers for (2) with $\mathcal{C}=\mathcal{X}$, note that (2) can be rewritten as the quadratic program (QP)

$$
\begin{aligned}
V_{N}^{\mathcal{X}}\left(x_{0}\right)= & \min _{\mathbf{u}_{N}} \frac{1}{2} \mathbf{u}_{N}^{T} H \mathbf{u}_{N}+x_{0}^{T} G \mathbf{u}_{N}+\frac{1}{2} x_{0}^{T} F x_{0} \\
\text { s.t } \quad & C \mathbf{u}_{N} \leq d-E x_{0},
\end{aligned}
$$

with appropriate matrices $H \in \mathbb{R}^{N \times N}, G \in \mathbb{R}^{n \times N}, F \in \mathbb{R}^{2 \times 2}$, $C \in \mathbb{R}^{6(N+1) \times N}, d \in \mathbb{R}^{6(N+1)}$ and $E \in \mathbb{R}^{6(N+1) \times n}$ (see [12] for details). It is easy to show that $\mathbf{u}_{N}^{*}$ is feasible for (23). Thus, 
to prove that $\mathbf{u}_{N}^{*}$ is the optimizer for (23), it is sufficient to find $\lambda \in \mathbb{R}^{6(N+1)}$ such that

$$
\begin{aligned}
H \mathbf{u}_{N}^{*}+G^{T} x_{0}+C^{T} \lambda & =0, \\
\lambda_{j} e_{j}^{T}\left(C \mathbf{u}_{N}^{*}+E x_{0}-d\right) & =0,
\end{aligned}
$$

and $\lambda_{j} \geq 0$ for every $j \in \mathbb{N}_{[1,6(N+1)]}$ (where $e_{j}$ is the $j$-th unit vector in $\mathbb{R}^{6(N+1)}$ ). It is easy to see that $C$ and $E$ (and accordingly d) can be chosen such that

$$
C \tilde{\mathbf{u}}_{N}+E x_{0}=\left(\begin{array}{llll}
\tilde{\mathbf{x}}_{N}^{T} & -\tilde{\mathbf{x}}_{N}^{T} & \tilde{\mathbf{u}}_{N}^{T} & -\tilde{\mathbf{u}}_{N}^{T}
\end{array}\right)^{T},
$$

where $\tilde{\mathbf{x}}_{N}$ is the state sequence associated with an (arbitrary) input sequence $\tilde{\mathbf{u}}_{N}$. According to (21), we have

$$
\pm u^{*}(k)<1, \quad x_{1}^{*}(k)=(-1)^{k} \cdot 5, \quad \text { and } \quad \pm x_{2}^{*}(k)<5
$$

for every $k \in \mathbb{N}_{[0, N-1]}$ and $k \in \mathbb{N}_{[0, N]}$, respectively. Thus, we find $e_{j}^{T}\left(C \mathbf{u}_{N}^{*}-d+E x_{0}\right)<0$ for every $j \in \mathcal{I}$, where

$$
\mathcal{I}:=\left\{j \in \mathbb{N}_{[1,6(N+1)]} \mid j \neq 2(N+1) \beta_{k}+4 k+1, k \in \mathbb{N}_{[0, N]}\right\}
$$

with $\beta_{k}:=k \bmod 2 \in\{0,1\}$. Analogously, we have $e_{j}^{T}\left(C \mathbf{u}_{N}^{*}-\right.$ $\left.d+E x_{0}\right)=0$ for every $j \in \mathcal{I}^{c}$, where the complement $\mathcal{I}^{c}$ is defined as $\mathcal{I}^{c}:=\mathbb{N}_{[1,6(N+1)]} \backslash \mathcal{I}$. The index set $\mathcal{I}$ obviously collects all inactive constraints. Analogously, $\mathcal{I}^{c}$ contains the indices of all weakly or strongly active constraints. Now, according to the complementary slackness conditions in (25), we obtain $\lambda_{j}:=0$ for every $j \in \mathcal{I}$. Without a proof, we claim that the choice $\lambda_{1}:=0$,

$$
\lambda_{2(N+1) \beta_{k}+4 k+1}:=\frac{513 \cdot 4^{N-k}-320 \cdot 2^{N-k}-63}{16 \cdot 2^{N-k}}
$$

for every $k \in \mathbb{N}_{[1, N-1]}$, and $\lambda_{2(N+1) \beta_{N}+4 N+1}:=\frac{153}{16}$ of the remaining Lagrange multipliers (with $j \in \mathcal{I}^{c}$ ) is such that the gradient condition (24) holds. Since the complementary slackness conditions are satisfied by construction and since it is easy to see that $\lambda_{j} \geq 0$ for every $j \in \mathbb{N}_{[1,6(N+1)]}, \mathbf{u}_{N}^{*}$ is indeed the optimizer for (23). As an immediate consequence, $\mathbf{u}_{N}^{*}$ and $\mathbf{x}_{N}^{*}$ are optimizers for (2) with $\mathcal{C}=\mathcal{X}$.

To prove that $x_{0} \in \mathcal{F}_{N}^{\mathcal{T}}$ (which is suggested by (20)), note that the sequences $\hat{\mathbf{u}}_{N}$ and $\hat{\mathbf{x}}_{N}$ with $\hat{u}(k):=u^{*}(k)$ for every $k \in$ $\mathbb{N}_{[0, N-2]}, \hat{x}(k):=x^{*}(k)$ for every $k \in \mathbb{N}_{[0, N-1]}$, and

$$
\hat{u}(N-1):=\frac{(-1)^{N-1} \cdot 5}{8} \text { and } \hat{x}(N):=(-1)^{N}\left(\begin{array}{r}
-\frac{9}{2} \\
\frac{5}{2}
\end{array}\right) \in \mathcal{T}
$$

are feasible (and in fact optimal) for (2) with $\mathcal{C}=\mathcal{T}$.

\section{An alternative proof of Theorem 3}

The following proof of Thm. 3 makes use of Lem. 1, which we state without proof (since it is trivial). In both statements, we frequently use the Minkowski function $\Psi_{\mathcal{C}}(x):=\inf \{\lambda \geq 0 \mid x \in$ $\lambda \mathcal{C}\}$ associated to a $\mathrm{C}$-set $\mathcal{C} \subset \mathbb{R}^{n}$.

Lemma 1: Let $\mathcal{T}$ satisfy Cond. 1 and let $\delta \in(0,1)$. Then there exists a finite $L \in \mathbb{N}_{[1, \infty)}$ such that

$$
\begin{aligned}
\Psi_{\mathcal{U}}\left(K(A+B K)^{k} x_{0}\right) & \leq \Psi_{\mathcal{T}}\left(x_{0}\right) \quad \forall k \in \mathbb{N}_{[0, L-1]}, \\
\Psi_{\mathcal{T}}\left((A+B K)^{k} x_{0}\right) & \leq \Psi_{\mathcal{T}}\left(x_{0}\right) \quad \forall k \in \mathbb{N}_{[0, L-1]}, \\
\Psi_{\mathcal{T}}\left((A+B K)^{L} x_{0}\right) & \leq \delta \Psi_{\mathcal{T}}\left(x_{0}\right)
\end{aligned}
$$

for every $x_{0} \in \mathbb{R}^{n}$.

Proof of Thm. 3. Choose $\psi \in \mathbb{R}_{+}$such that

$$
\psi \geq \max _{x \in \mathcal{F}_{\infty}^{\mathcal{X}}} \Psi_{\mathcal{T}}(x)
$$

and note that $\psi \geq 1$ due to $\mathcal{T} \subseteq \mathcal{F}_{\infty}^{\mathcal{X}}$. Further note that (29) implies $\frac{1}{\psi} \mathcal{F}_{\infty}^{\mathcal{X}} \subseteq \mathcal{T}$. Thus, (11) trivially holds for every $\lambda \in\left(0, \frac{1}{\psi}\right]$ and every $N \in \mathbb{N}$ since we have $\mathcal{T} \subseteq \mathcal{S}_{k}^{c}$ for every $k \in \mathbb{N}$ according to Thm. 1 .
It remains to prove (11) for $\lambda \in\left(\frac{1}{\psi}, 1\right)$ and some $N \in \mathbb{N}$. Clearly, the interval $\left(\frac{1}{\psi}, 1\right)$ is empty for $\psi=1$ (which immediately completes the proof for this case). We consequently assume $\psi>$ 1 in the following. Now, consider $\delta=\frac{1}{\psi^{2}}$ and let $L \in \mathbb{N}_{[1, \infty)}$ be such that (26)-(28) hold (existence is guaranteed by Lem. 1). Choose any $x_{0} \in \lambda \mathcal{F}_{\infty}^{\mathcal{X}}$. Since $\mathcal{F}_{\infty}^{\mathcal{X}}$ is controlled invariant, there exist inputs $\tilde{u}(0), \ldots, \tilde{u}(L-1) \in \lambda \mathcal{U}$ such that the associated states $\tilde{x}(1), \ldots, \tilde{x}(L)$ satisfy $\tilde{x}(k) \in \lambda \mathcal{F}_{\infty}^{\mathcal{X}} \subseteq \lambda \mathcal{X}$ for every $k \in \mathbb{N}_{[1, L]}$. Next, let

$$
\alpha_{1}:=\frac{\frac{1}{\lambda}-1}{\psi-1} \quad \text { and } \quad \mu_{1}:=\left(1+\frac{1}{\psi}\right) \lambda-\frac{1}{\psi},
$$

note that $\alpha_{1} \in(0,1)$ and $\mu_{1} \in(0, \lambda)$, and consider the inputs

$$
\hat{u}(k):=\left(1-\alpha_{1}\right) \tilde{u}(k)+\alpha_{1} K(A+B K)^{k} x_{0} .
$$

and the associated states

$$
\hat{x}(k):=\left(1-\alpha_{1}\right) \tilde{x}(k)+\alpha_{1}(A+B K)^{k} x_{0} .
$$

It is easy to show that we have

$$
\max \left\{\Psi_{\mathcal{U}}(\hat{u}(k)), \Psi_{\mathcal{X}}(\hat{x}(k))\right\} \leq\left(1-\alpha_{1}\right) \lambda+\alpha_{1} \psi \lambda=1
$$

or, equivalently, $\hat{u}(k) \in \mathcal{U}$ and $\hat{x}(k) \in \mathcal{X}$ for every $k \in \mathbb{N}_{[0, L-1]}$. Moreover, we find

$$
\Psi_{\mathcal{F} \mathcal{X}}(\hat{x}(L)) \leq\left(1-\alpha_{1}\right) \lambda+\alpha_{1} \delta \psi \lambda=\mu_{1}
$$

or, equivalently, $\hat{x}(L) \in \mu_{1} \mathcal{F}_{\infty}^{\mathcal{X}}$. In other words, every $x_{0} \in \lambda \mathcal{F}_{\infty}^{\mathcal{X}}$ can be steered to $\mu_{1} \mathcal{F}_{\infty}^{\mathcal{X}} \subset \lambda \mathcal{F}_{\infty}^{\mathcal{X}}$ using an input sequence of length $L$. The described procedure can be easily repeated. Thereby, the closer we get to the origin, the interpolation via $\alpha_{j}$ can be adjusted in favor of the converging unconstrained trajectory. In fact, defining $\mu_{0}:=\lambda$, (30) can be easily generalized in terms of

$$
\alpha_{j}:=\frac{\frac{1}{\mu_{j-1}}-1}{\psi-1} \text { and } \mu_{j}:=\left(1+\frac{1}{\psi}\right) \mu_{j-1}-\frac{1}{\psi}
$$

for every $j \in \mathbb{N}_{[1, \infty)}$. This means that every $x_{0} \in \lambda \mathcal{F}_{\infty}^{\mathcal{X}}$ can be steered to $\mu_{j} \mathcal{F}_{\infty}^{\mathcal{X}}$ using an input sequence of length $j L$. Obviously, if $\mu_{j} \leq \frac{1}{\psi}$ for some $j \in \mathbb{N}_{[1, \infty)}$, we have $\mu_{j} \mathcal{F}_{\infty}^{\mathcal{X}} \subseteq \mathcal{T}$ and thus $\hat{x}(j L) \in \mathcal{T}$. Recognizing that $\mu_{j}$ can be explicitly computed as $\mu_{j}=1-(1-\lambda)\left(1+\frac{1}{\psi}\right)^{j}$, it is easy to show that the smallest step $j$ such that $\mu_{j} \leq \frac{1}{\psi}$ evaluates to

$$
J:=\min \left\{j \in \mathbb{N} \mid \mu_{j} \leq \frac{1}{\psi}\right\}=\left\lceil\ln \left(\frac{1-\frac{1}{\psi}}{1-\lambda}\right) \ln \left(1+\frac{1}{\psi}\right)^{-1}\right\rceil .
$$

Now, an $N \in \mathbb{N}$ such that (11) holds can be chosen analogously to the proof of Thm. 4. In fact, any $N$ satisfying (15) with $M=J L$ is appropriate.

Remark 1: The proof of Thm. 3 is similar to but different from the proofs of [6, Prop. 12 and Thm. 13]. The main difference to the proofs in [6] is that we make use of Lem. 1 and that we adjust the interpolation according to (31). As a consequence, an upper bound for an $N \in \mathbb{N}$ that satisfies (15) can be written as

$$
N<a+b \ln \left(\frac{1}{1-\lambda}\right)
$$

for some $a, b \in \mathbb{R}_{+}$, while the proof of [6, Prop. 12] only guarantees $N<c+\frac{d}{1-\lambda}$ for some $c, d \in \mathbb{R}_{+}$(cf. [6, Eq. (20)]). Obviously, $\ln \left(\frac{1}{1-\lambda}\right) \ll \frac{1}{1-\lambda}$ for $\lambda \rightarrow 1$. To reproduce (32), first note that there exists an $N \in \mathbb{N}$ satisfying (15) with $M=J L$ such that

$$
N<\frac{1}{\underline{q}}(\bar{p}-\underline{p}+(J L-1) \bar{q}+J L \bar{r})+2 .
$$

Now, using the overestimation

$$
J<\frac{\ln \left(\frac{1}{1-\lambda}\right)+\ln (\psi-1)-\ln (\psi)}{\ln (\psi+1)-\ln (\psi)}+1,
$$

it is easy to derive $a$ and $b$ in (32). 